\title{
End-functionalized glycopolymers as mimetics of chondroitin sulfate proteoglycans $\dagger$
}

\author{
Song-Gil Lee, $\$$ Joshua M. Brown, $\$$ Claude J. Rogers, John B. Matson, Chithra Krishnamurthy, Manish Rawat \\ and Linda C. Hsieh-Wilson*
}

\author{
Received 18th April 2010, Accepted 15th July 2010 \\ DOI: 10.1039/c0sc00271b
}

\begin{abstract}
Glycosaminoglycans are sulfated polysaccharides that play important roles in fundamental biological processes, such as cell division, viral invasion, cancer and neuroregeneration. The multivalent presentation of multiple glycosaminoglycan chains on proteoglycan scaffolds may profoundly influence their interactions with proteins and subsequent biological activity. However, the importance of this multivalent architecture remains largely unexplored, and few synthetic mimics exist for probing and manipulating glycosaminoglycan activity. Here, we describe a new class of end-functionalized ring-opening metathesis polymerization (ROMP) polymers that mimic the native-like, multivalent architecture found on chondroitin sulfate (CS) proteoglycans. We demonstrate that these glycopolymers can be readily integrated with microarray and surface plasmon resonance technology platforms, where they retain the ability to interact selectively with proteins. ROMP-based glycopolymers are part of a growing arsenal of chemical tools for probing the functions of glycosaminoglycans and for studying their interactions with proteins.
\end{abstract}

\section{Introduction}

Carbohydrates possess greater structural diversity than either nucleic acids or proteins. Although they participate in a wide range of critical processes and alterations in their structure have been linked to a number of human diseases, they remain underexplored targets for chemical biology and pharmaceutical chemistry. We have embarked on a program to study a large class of sulfated polysaccharides known as glycosaminoglycans, with the goals of understanding their structure-function relationships and gaining insight into the molecular mechanisms underlying their biological activity.

Glycosaminoglycans (GAGs) are polymers, composed of 10-200 repeating sulfated disaccharide units (Fig. 1A). ${ }^{1}$ GAGs contain regions of high and low sulfation, ${ }^{2}$ with highly sulfated regions serving as binding sites for proteins. ${ }^{3}$ These protein interactions endow GAGs with the ability to regulate essential processes such as cell division, viral invasion, blood coagulation and neuronal regeneration. ${ }^{1 a, b, 3 a, 4}$ GAGs are covalently attached to various proteoglycan proteins, with some proteoglycans bearing as many as 100 sugar chains (Fig. 1B). ${ }^{5}$ It has been established using synthetic glycopolymers and oligosaccharides for other systems that the relative position and density of sugars can impact the avidity and specificity of glycan-protein interactions. ${ }^{6}$ However, despite these important advances, the role of the multivalent architecture found in native GAG structures has remained largely unexplored.

Division of Chemistry and Chemical Engineering and Howard Hughes Medical Institute, California Institute of Technology, Pasadena, California, 91125, USA. E-mail: Ihw@caltech.edu; Fax: +1 626564 9297; Tel: +16263956101

$\dagger$ Electronic supplementary information (ESI) available: Experimental procedures and compound characterizations. See DOI: $10.1039 / \mathrm{c} 0 \mathrm{sc} 00271 \mathrm{~b}$

\$ These authors equally contributed to this work.
A

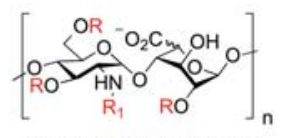

heparan sulfate/heparin

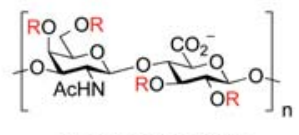

chondroitin sulfate
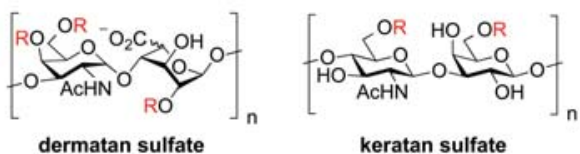

keratan sulfate

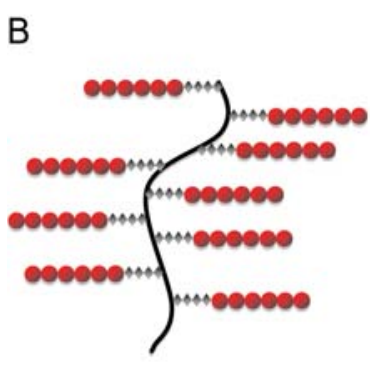

C

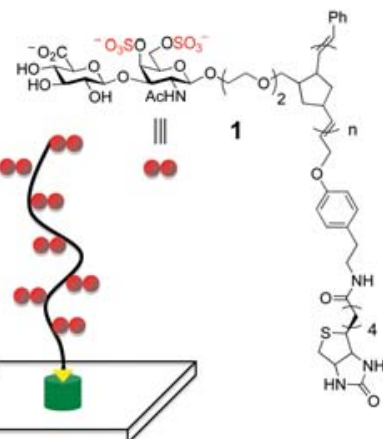

Fig. 1 (A) Structures of representative GAG classes. $\mathrm{R}=\mathrm{SO}_{3}{ }^{-}$or $\mathrm{H}$; $\mathrm{R}_{1}=\mathrm{SO}_{3}{ }^{-}, \mathrm{H}$, or Ac; $n=\sim 10-200$. (B) Schematic representation of a proteoglycan, which typically consists of multiple GAG chains attached to a protein core. (C) Biotin end-functionalized ROMP polymers as mimetics of CS proteoglycans. $n=\sim 80-280$.

Here, we describe a new class of end-functionalized GAG mimetic glycopolymers that are designed to mimic the multivalent presentation of chondroitin sulfate (CS) on proteoglycans. We demonstrate that these glycopolymers can be integrated with microarray as well as surface plasmon resonance (SPR) technology platforms to probe GAG-protein interactions and study the activity of specific sulfation motifs. 


\section{Results and discussion}

To mimic the orientation of the sugar chains on proteoglycans, we designed glycopolymer $\mathbf{1}$, which has an end-functionalized biotin moiety to achieve the desired orientation of the pendant sugars and to facilitate attachment of the polymer to surfaces (Fig. 1C). We chose a norbornene-based backbone to allow for multivalent display of the sugar chains at defined, chemically controlled intervals and to confer a degree of rigidity to the structure. Previous studies in our laboratory have demonstrated that glycopolymers containing complex, highly anionic di- and tetrasaccharides can be generated using ring-opening metathesis polymerization (ROMP) chemistry, although more flexible ciscyclooctene monomers were employed. ${ }^{7}$ In addition to increasing the structural rigidity of the resultant polymer, norbornenebased monomers would have the advantage of enabling access to block copolymers for controlling the sulfation motifs between GAG chains.

Glycopolymer 2 was synthesized from monomer 3, which contains the biologically active CS-E disaccharide unit (Scheme 1). ${ }^{8}$ Briefly, trichloroacetimidate donor $\mathbf{4}$ was coupled to norbornene acceptor 5 to provide fully protected disaccharide $\mathbf{6}$ in good yield and with excellent $\beta$-stereoselectivity. Radical-mediated conversion of the $N$-trichloroacetyl group to an $N$-acetyl group and DDQ oxidation of the $p$-methoxybenzylidene acetal afforded diol 7. Sulfation using sulfur trioxide trimethylamine complex afforded the desired norbornene monomer 3 in $83 \%$ yield. A major challenge for the polymerization reaction was the low solubility of sulfated oligosaccharides in the non-coordinating, aprotic solvents typically used for ROMP. Fortunately, the fully protected, sulfated monomer 3 was soluble in $\mathrm{MeOH} /$ $\left(\mathrm{CH}_{2} \mathrm{Cl}\right)_{2}$ co-solvent mixtures, and polymerization with $1.0 \mathrm{~mol} \%$ of Grubbs' catalyst $\left(\mathrm{H}_{2} \mathrm{IMes}\right)(\mathrm{Py})_{2}(\mathrm{Cl})_{2} \mathrm{Ru}=\mathrm{CHPh}(\mathbf{8})^{9}$ led to complete conversion to the desired glycopolymer 9 within $5 \mathrm{~min}$ (Table 1, degree of polymerization $(\mathrm{DP})=97$; polydispersity index $(\mathrm{PDI})=1.17)$. Lowering the catalyst concentration to $0.5 \mathrm{~mol} \%$ produced glycopolymer $\mathbf{1 0}$ with exceptionally long chain lengths $(\mathrm{DP}=281$; PDI $=1.07)$. Longer polymers with narrower polydispersities were attainable with norbornene

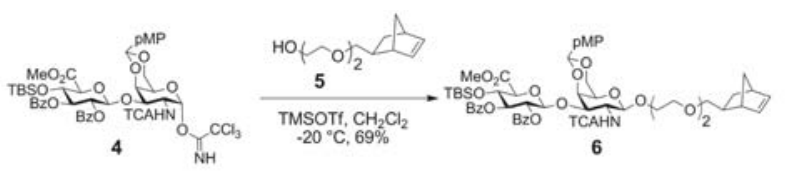

1. $\mathrm{Bu} \mathrm{S}_{3} \mathrm{SnH}, \mathrm{AlBN}$, benzene 1. $\mathrm{Bu}_{3} \mathrm{SnH}$, AlBN, benzene
$80^{\circ} \mathrm{C}, 3 \mathrm{~h}, 77 \%$
2. $\mathrm{DDQ}, \mathrm{CH}_{3} \mathrm{CN} / \mathrm{H}_{2} \mathrm{O}$
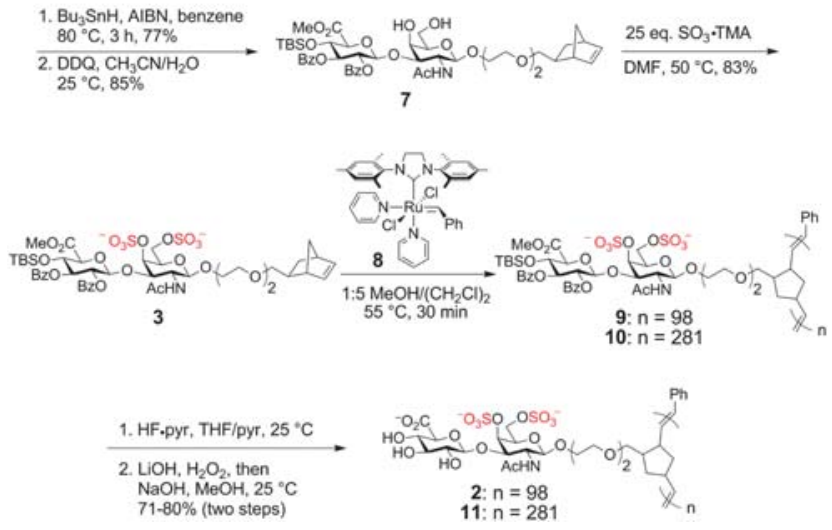

Scheme 1 Synthesis of Norbornene-based CS Glycopolymers
Table 1 Generation of CS Glycomimetics Using ROMP

\begin{tabular}{|c|c|c|c|c|c|c|c|}
\hline entry & monomer & $\begin{array}{l}\text { mol \% } \\
\text { catalyst }\end{array}$ & polymer & $\mathrm{n}$ (DP) & $\%$ yield & $\begin{array}{l}\mathrm{M}_{\mathrm{n}}{ }^{a} / \mathrm{g} \\
\mathrm{mol}^{-1}\end{array}$ & PDI \\
\hline 1 & 3 & 1.0 & 9 & 97 & 87 & 105,100 & 1.17 \\
\hline 2 & 3 & 0.5 & 10 & 281 & 92 & 283,100 & 1.07 \\
\hline 3 & 3 & 1.0 & 1 & 86 & $74^{b}$ & 68,480 & 1.05 \\
\hline 4 & 7 & 1.0 & 15 & 82 & $55^{b}$ & 48,480 & 1.10 \\
\hline
\end{tabular}

${ }^{a}$ Number average molecular weight and polydispersity index were determined by GPC (0.2 M LiBr in DMF for entries $1-2 ; 100 \mathrm{mM}$ $\mathrm{NaNO}_{3}$ and $200 \mathrm{ppm} \mathrm{NaN}$ in water for entries 3-4). ${ }^{b}$ Yield for 3 steps (polymerization, desilylation, saponification).

relative to cis-cyclooctene monomers, ${ }^{7,10}$ which may be advantageous for biological activity. The CS glycomimetics $\mathbf{2}$ and $\mathbf{1 1}$ were obtained after desilylation and sequential $\mathrm{LiOOH}-\mathrm{NaOH}$ treatment in $71-80 \%$ yield over two steps.

We next investigated end-functionalization of the glycopolymers with a biotin moiety. Previous studies have established that internal cis-olefin or enol ether terminating agents (TA) can be used for the direct, efficient capping of ROMP polymers. ${ }^{11}$ Addition of the biotin terminating agent $\mathbf{1 2}^{\mathbf{1 1 b}}$ to the reaction mixture after completion of the living polymerization, resulted in the desired end-capping of the glycopolymer to produce 13 (Scheme 2). Desilylation and saponification afforded glycopolymer 1 in $74 \%$ overall yield over the 3 steps (Table 1, $\mathrm{DP}=86$; PDI $=1.05$ ). The end-capping efficiency of $20 \%$, as determined using a colorimetric biotin quantification assay and ${ }^{1} \mathrm{H}$ NMR, was modest due to the limited solubility of the unquenched glycopolymer and 12. For comparison, we also synthesized the biotin-functionalized unsulfated glycopolymer 15 $(\mathrm{DP}=82$; PDI $=1.10)$ from monomer 7 using a similar approach.

To explore the ability of the glycopolymers to interact with protein receptors, glycopolymers $\mathbf{1}$ and $\mathbf{1 5}$ were attached to microarray surfaces. A high-precision contact-printing robot was used to deliver nanoliter volumes of the biotin-labeled glycopolymers to streptavidin-coated slides, yielding spots approximately $200 \mu \mathrm{m}$ in diameter. We examined the binding of monoclonal antibodies 2D11 and 2D5, which are selective for the CS-E and CS-C sulfation motifs, respectively. ${ }^{3 b, 8 a}$ The microarrays were incubated with each antibody $(70 \mathrm{nM})$, and protein binding was visualized using a secondary $\mathrm{Cy} 3$-conjugated goat anti-mouse antibody. Antibody 2D11 bound selectively to CS-E sulfated glycopolymer 1 and showed no detectable binding to
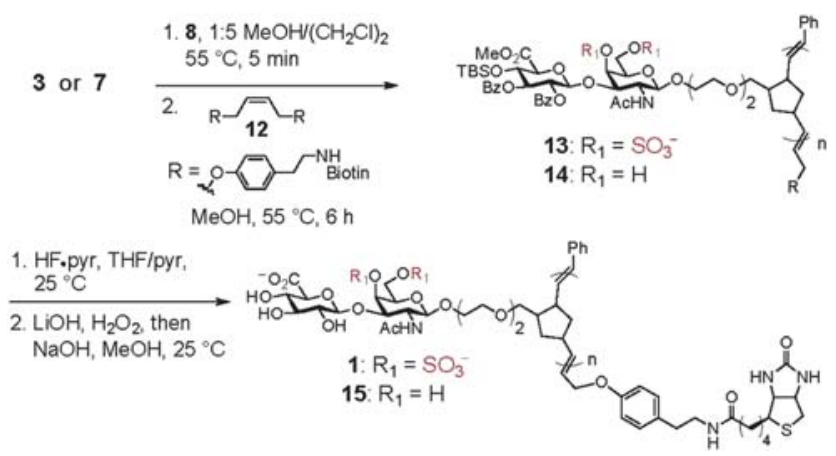

Scheme 2 Synthesis of Biotin End-Functionalized CS Glycopolymers 


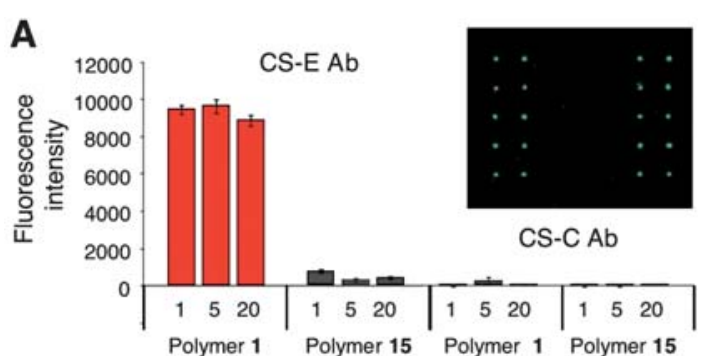

[Glycopolymer] $(\mu \mathrm{M})$

B

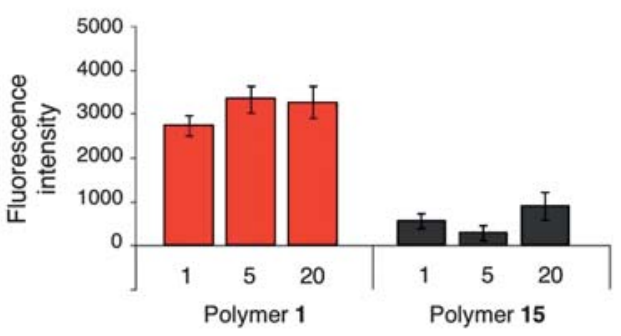

[Glycopolymer] $(\mu \mathrm{M})$

Fig. 2 (A) Binding of the CS-E Ab (left) and CS-C Ab (right) to CS-E glycopolymer 1 and unsulfated glycopolymer 15 immobilized on microarrays. (B) Binding of GDNF to glycopolymers 1 and 15. Each microarray contained 640 spots, and the bar graphs represent the quantification for selected concentrations of glycopolymer (1280 data points per protein). The inset shows binding of the CS-E Ab to 1 on a representative portion of the array. See Supplementary Information $\dagger$ for details.

unsulfated glycopolymer 15 (Fig. 2A). Moreover, no binding of the CS-C antibody 2D5 to either glycopolymer was observed, consistent with the selective recognition of specific sulfated epitopes. We also examined the binding of several growth factors, including glial cell-derived neurotrophic factor (GDNF), a growth factor important for the survival and differentiation of dopaminergic neurons. ${ }^{12}$ Although the binding of GDNF to a highly sulfated mixture of chondroitin and dermatan sulfate chains has been studied, ${ }^{3 c}$ its ability to recognize homogeneous, well-defined CS structures has not been explored. Significant binding of GDNF to the CS-E glycopolymer, but not the unsulfated glycopolymer, was observed (Fig. 2B), indicating a clear preference of GDNF for the sulfated sugar epitope.

Finally, we investigated whether the end-functionalized glycopolymers could be used to facilitate quantitative, real-time analysis of GAG-protein interactions using surface plasmon resonance (SPR). Glycopolymers $\mathbf{1}$ and $\mathbf{1 5}$ were immobilized on streptavidin-conjugated CM5 sensor chips at low density $\left(\mathrm{R}_{\mathrm{L}} \approx\right.$ $25 \mathrm{RU}$ ) to prevent mass transfer-limited kinetics. Binding of GDNF to the glycopolymers was assessed by flowing GDNF over the chip at various concentrations $(2,1,0.5 \mathrm{nM})$ and recording the SPR sensorgrams $\left(50 \mu \mathrm{L} \mathrm{min}{ }^{-1}, 25{ }^{\circ} \mathrm{C}\right)$. As shown in Fig. 3, GDNF interacted with the CS-E sulfated glycopolymer, but not with the unsulfated glycopolymer, consistent with the microarray results. Binding of GDNF to glycopolymer $\mathbf{1}$ was characterized by a slow initial rate of association that rapidly reached equilibrium, followed by a slow rate of dissociation. By plotting the response at equilibrium for varying concentrations of GDNF $(0.25-62 \mathrm{nM})$, we obtained a dissociation constant $\left(K_{D}\right)$ of $6 \pm$ $1 \mathrm{nM}$ for the interaction between GDNF and glycopolymer 1 . It is
A

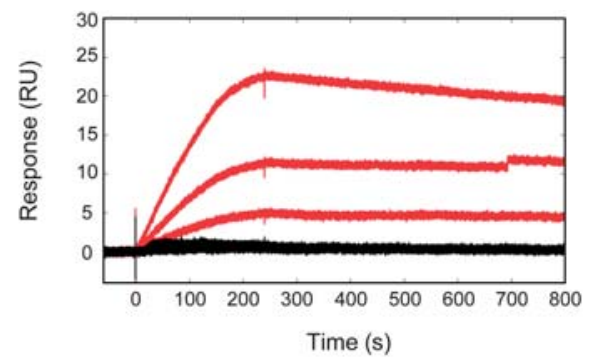

B

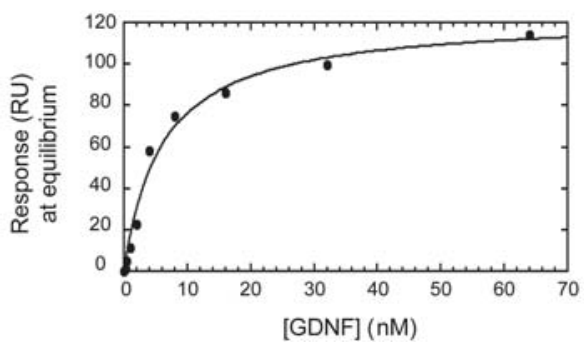

Fig. 3 Surface plasmon resonance of GDNF binding to CS glycopolymers 1 and 15. GDNF at varying concentrations $(2,1$, and $0.5 \mathrm{nM})$ binds to the CS-E sulfated glycopolymer (red), but not the unsulfated polymer (black). (B) The dissociation constant $\left(K_{D}\right)$ for the interaction between 1 and GDNF was measured by plotting the response at equilibrium for varying concentrations of GDNF. Nonlinear regression analysis gave a $K_{D}$ of $6 \pm 1 \mathrm{nM}$.

well known that monovalent CS and heparan sulfate disaccharides exhibit weak binding affinity for proteins and minimal biological activity. ${ }^{7,8 b, 13}$ Indeed, binding of GDNF to a biotinylated CS-E disaccharide could not be detected under these conditions (Fig. S2 $\dagger$ ). Thus, the observation that our glycopolymers interact strongly with proteins suggests that the multivalent display of sulfated epitopes between GAG chains plays a critical role in enhancing their interactions with proteins. Together, our studies demonstrate that end-functionalized ROMP glycopolymers can effectively engage glycosaminoglycan-binding proteins and function as novel mimetics for CS glycosaminoglycans.

\section{Conclusions}

We have generated a new class of CS glycomimetic polymers that display defined sulfation motifs, while mimicking the multivalent architecture of native GAG chains. Our studies demonstrate that these glycopolymers can be efficiently attached to surfaces, where they approximate physiological cell-cell and cell-extracellular matrix interactions and retain the ability to engage proteins. ROMP-based glycopolymers are part of a growing arsenal of chemical tools for studying the structure-activity relationships of GAGs. We anticipate that they will prove valuable for understanding how multivalency, not only within but also between, GAG chains enhances the avidity, specificity and cooperativity of GAG-protein interactions. Future studies will focus on extending the methodology reported herein to polymers and block copolymers with varied sulfation patterns and applying them as tools to manipulate $\mathrm{CS}$ activity in various biological contexts.

\section{Notes and references}

1 (a) I. Capila and R. J. Linhardt, Angew. Chem. Int. Ed. Engl., 2002, 41, 390; (b) K. Sugahara, T. Mikami, T. Uyama, S. Mizuguchi, K. Nomura 
and H. Kitagawa, Curr. Opin. Struct. Biol., 2003, 13, 612; (c) C. I. Gama and L. C. Hsieh-Wilson, Curr. Opin. Chem. Biol., 2005, 9, 609.

2 (a) H. Desaire, T. L. Sirich and J. A. Leary, Anal. Chem., 2001, 73, 3513; (b) J. E. Turnbull and J. T. Gallagher, Biochem. J., 1991, 273(Pt 3), 553

3 (a) R. Raman, V. Sasisekharan and R. Sasisekharan, Chem. Biol., 2005, 12, 267; (b) S. E. Tully, M. Rawat and L. C. Hsieh-Wilson, J. Am. Chem. Soc., 2006, 128, 7740; (c) C. D. Nandini, N. Itoh and K. Sugahara, J. Biol. Chem., 2005, 280, 4058; (d) S. S. Deepa, Y. Umehara, S. Higashiyama, N. Itoh and K. Sugahara, J. Biol. Chem., 2002, 277, 43707; (e) E. L. Shipp and L. C. Hsieh-Wilson, Chem. Biol., 2007, 14, 195.

4 (a) S. Mizuguchi, T. Uyama, H. Kitagawa, K. H. Nomura, K. Dejima, K. Gengyo-Ando, S. Mitani, K. Sugahara and K. Nomura, Nature, 2003, 423, 443; (b) J. M. Trowbridge and R. L. Gallo, Glycobiology, 2002, 12, 117R; (c) Y. Shen, A. P. Tenney, S. A. Busch, K. P. Horn, F. X. Cuascut, K. Liu, Z. He, J. Silver and J. G. Flanagan, Science, 2009, 326, 592.

5 L. Kjellen and U. Lindahl, Annu. Rev. Biochem., 1991, 60, 443.

6 (a) D. M. Lewallen, D. Siler and S. S. Iyer, ChemBioChem, 2009, 10 , 1486; (b) J. E. Gestwicki, C. W. Cairo, L. E. Strong, K. A. Oetjen and L. L. Kiessling, J. Am. Chem. Soc., 2002, 124, 14922; (c) N. Horan, L. Yan, H. Isobe, G. M. Whitesides and D. Kahne, Proc. Natl. Acad. Sci. U. S. A., 1999, 96, 11782; (d) M. Dhayal and D. M. Ratner, Langmuir, 2009, 25, 2181; (e) H. M. Branderhorst R. Ruijtenbeek, R. M. Liskamp and R. J. Pieters, ChemBioChem, 2008, 9, 1836; (f) K. Godula, D. Rabuka, K. T. Nam and C. R. Bertozzi, Angew. Chem., Int. Ed., 2009, 48, 4973; (g) J. E. Gestwicki, C. W. Cairo, D. A. Mann, R. M. Owen and
L. L. Kiessling, Anal. Biochem., 2002, 305, 149; (h) J. Geng, G. Mantovani, L. Tao, J. Nicolas, G. Chen, R. Wallis, D. A. Mitchell, B. R. G. Johnson, S. D. Evans and D. M. Haddleton, J. Am. Chem. Soc., 2007, 129, 15156; (i) K. Matsuura, H. Kitakouji, N. Sawada, H. Ishida, M. Kiso, K. Kitajima and K. Kobayashi, J. Am. Chem. Soc., 2000, 122, 7406.

7 M. Rawat, C. I. Gama, J. B. Matson and L. C. Hsieh-Wilson, J. Am. Chem. Soc., 2008, 130, 2959.

8 (a) C. I. Gama, S. E. Tully, N. Sotogaku, P. M. Clark, M. Rawat, N. Vaidehi, W. A. Goddard, 3rd, A. Nishi and L. C. Hsieh-Wilson, Nat. Chem. Biol., 2006, 2, 467; (b) S. E. Tully, R. Mabon, C. I. Gama, S. M. Tsai, X. Liu and L. C. Hsieh-Wilson, J. Am. Chem. Soc., 2004, 126, 7736.

9 (a) K. D. Camm, N. M. Castro, Y. Liu, P. Czechura, J. L. Snelgrove and D. E. Fogg, J. Am. Chem. Soc., 2007, 129, 4168; (b) J. A. Love, J. P. Morgan, T. M. Trnka and R. H. Grubbs, Angew. Chem., Int. Ed., 2002, 41, 4035 .

10 R. Walker, R. M. Conrad and R. H. Grubbs, Macromolecules, 2009 , 42, 599.

11 (a) J. B. Matson and R. H. Grubbs, Macromolecules, 2008, 41, 5626; (b) J. B. Matson and R. H. Grubbs, Macromolecules, 2010, 43, 213; (c) R. M. Owen, J. E. Gestwicki, T. Young and L. L. Kiessling, Org. Lett., 2002, 4, 2293.

12 (a) L. F. Lin, D. H. Doherty, J. D. Lile, S. Bektesh and F. Collins, Science, 1993, 260, 1130; (b) A. Tomac, E. Lindqvist, L. F. Lin, S. O. Ogren, D. Young, B. J. Hoffer and L. Olson, Nature, 1995, 373, 335 .

13 J. L. de Paz, C. Noti, F. Bohm, S. Werner and P. H. Seeberger, Chem. Biol., 2007, 14, 879. 